\title{
Even-odd behavior of conductance in monatomic sodium wires
}

\author{
H.-S. Sim,,$^{1,2}$ H.-W. Lee, ${ }^{3}$ and K. J. Chang ${ }^{1,3}$ \\ ${ }^{1}$ Department of Physics, Korea Advanced Institute of Science and Technology, Taejon 305-701, Korea \\ ${ }^{2}$ Max-Planck-Institute for the Physics of Complex Systems, Nöthnitzer Str. 38, D-01187 Dresden, Germany \\ ${ }^{3}$ School of Physics, Korea Institute for Advanced Study, 207-43 Cheongryangri-dong, Dongdaemun-gu, Seoul 130-012, Korea
}

(November 21, 2018)

\begin{abstract}
With the aid of the Friedel sum rule, we perform first-principles calculations of conductances through monatomic $\mathrm{Na}$ wires, taking into account the sharp tip geometry and discrete atomic structure of electrodes. We find that conductances $(G)$ depend on the number $(L)$ of atoms in the wires; $G$ is $G_{0}\left(=2 e^{2} / h\right)$ for odd $L$, independent of the wire geometry, while $G$ is generally smaller than $G_{0}$ and sensitive to the wire structure for even $L$. This even-odd behavior is attributed to the charge neutrality and resonant character due to the sharp tip structure. We suggest that similar even-odd behavior may appear in other monovalent atomic wires.
\end{abstract}

Atomic contacts have been generated by scanning tunneling microscopes or mechanically controllable breakjunctions [1]. It has been demonstrated that single atom contacts made of monovalent atoms such as $\mathrm{Na}$, $\mathrm{K}$, and $\mathrm{Au}$ have a strong tendency towards the quantized conductance $G_{0}\left(=2 e^{2} / h\right)$. To explain the quantization, a ballistic transport model has been suggested [2, where atomic contacts are modeled by jellium constrictions, which are adiabatically connected to electrodes, analogous to quantum point contacts (QPC) in twodimensional electron gas systems [3, 迎. However, this analogy may be inappropriate for sharp tip structures of atomic contacts [5], because the Fermi wavelengths in metallic systems are comparable to atomic spacings. Recently, Yeyati and his coworkers [6] have suggested a resonant transport as an alternative explanation, based on their tight-binding calculations assuming the local charge neutrality. Despite several theoretical attempts [6] 9], the origin of the quantized conductance has not been clearly understood because the validity of simplifications such as the local charge neutrality assumption [6.8] or a jellium electrode model [9], which ignores the sharp tip geometry for electrodes, is rather unclear.

In this Letter, to clarify the origin of the conductance quantization, we calculate conductances through monatomic Na wires [10], taking into account the sharp tip shape and discrete atomic structure of electrodes. We perform real-space multigrid electronic structure calculations within the local-density-functional approximation (LDA). Using the Friedel sum rule [11,12], which relates the conductance to the density of states (DOS), we are able to reduce greatly computational demands. We find a resonant character in transport and a robust quantization of conductances when the number $(L)$ of atoms in wires is odd. For even $L$, conductances are not quantized, resulting in even-odd behavior in transport. This feature is very different from the length-independent conductances observed in ballistic quantum wires.

Figure [1(a) shows a monatomic Na wire connected to two electrodes, which have an inversion symmetry. Each electrode is modeled by a cluster of $M \mathrm{Na}$ atoms in the bcc structure. The (111) direction of the bcc lattice is aligned with the wire with $L \mathrm{Na}$ atoms, which consists of 2 apex atoms in the clusters and $L-2 \mathrm{Na}$ atoms between the clusters. For the interatomic spacing $d$ within the wire, we use the bond distance of $d_{0}=3.659 \AA$ in bcc bulk $\mathrm{Na}$, and also test various different values for $d$. Using the inversion symmetry, the DOS can be decomposed into even $\left(\rho_{e}\right)$ and odd parity $\left(\rho_{o}\right)$ components. When only one eigenchannel contributes to transport [13,9], conductances through the wire can be expressed in terms of $\rho_{e}$ and $\rho_{o}$ using the Friedel sum rule [11,12];

$$
G=\frac{2 e^{2}}{h} \sin ^{2}\left[\frac{\pi}{2}\left(N_{e}-N_{o}\right)\right]
$$

where $N_{e(o)}\left[=\int^{E_{F}} d E \rho_{e(o)}(E)\right]$ denotes the number of electrons with even (odd) parity and $E_{F}$ is the Fermi energy. With Eq. (11), we can reduce greatly the computational demand and analyze conductance characteristics in terms of the DOSs.

We calculate self-consistently the electronic energies of the wire system using the real-space multigrid method [14 within the LDA. Norm-conserving pseudopotentials are generated by the scheme of Troullier and Martins, and then transformed into the separable form of Kleinman and Bylander 15]. We use a supercell geometry containing two electrodes, each of which has a cross section of $20.76 \AA$ wide. Electrodes in neighboring supercells are separated by more than $11.64 \AA$, so that inter-supercell interactions are negligible. Using a grid spacing of 0.43 $\AA$, we ensure that the total energy is converged to within $10^{-7}$ Ry. To calculate the DOS, we use the Fermi-Dirac broadening of $E_{T}=0.052 \mathrm{eV}(600 \mathrm{~K})$ for the occupation of each level. We find that the level splitting between even and odd parity states, which have negligible wire characteristics, is less than $0.003 \mathrm{eV}$. Since this value is much smaller than $E_{T}$ and the average level spacing near 
$E_{F}$, we do not expect numerical errors in $N_{e}-N_{o}$ due to the charge sloshing problem [16].

The conductances calculated from the DOSs are plotted as a function of $L$ for $M=64$ and 95 in Fig. 2. In both cases $G$ oscillates with $L$, exhibiting even-odd behavior. For odd $L$, we obtain $G=G_{0}$ since the difference $\left(N_{e}-N_{o}\right)$ almost equals 1 . For both $M=64$ and 95, similar results are found, indicating that the finite size effect of clusters is negligible for odd $L$. For even $L$, on the other hand, $N_{e}-N_{o}$ is not equal to 1 and conductances deviate considerably from $G_{0}$. Since the results for $M=64$ and 95 are different from each other, the finite size effect seems to be more important for even $L$. At this point, it is beyond our computational capability to calculate conductances for larger cluster sizes. However, based on the DOS analysis given below, we confirm that the qualitative feature of $G$ being smaller than $G_{0}$ for even $L$ is not an artifact due to the finite size effect.

The DOS analysis provides useful information. From 95-atom cluster calculations, the results for $\rho_{e}(E)$ and $\rho_{o}(E)$ are drawn for the $L=4$ and 5 wires in Fig. 3, and compared with $\rho_{e}^{\mathrm{R}}(E)$ and $\rho_{o}^{\mathrm{R}}(E)$ for their reference systems (hereafter denoted by the superscript $R$ ), which are defined by removing $L-2$ central atoms in the wire without altering the inter-cluster distance. We find that $\rho_{e(o)}$ and $\rho_{e(o)}^{\mathrm{R}}$ are almost identical over a wide range of energies except for near the Fermi level. From the projected DOSs (PDOS) onto the $L-2$ central atoms in the wire (here a sphere with the radius of $0.5 d$ is chosen for each atom), the difference between $\rho_{e(o)}(E)$ and $\rho_{e(o)}^{\mathrm{R}}(E)$ is found to be mainly caused by the existence of resonance states. Thus, unlike conventional QPCs, the monatomic wires have the resonant character, consistent with previous results [6].

We estimate the spatial extension of the resonance states by comparing the charge densities of the wire system $(L=5, M=64)$ with those for its reference system [see Fig. [1(b)]. Besides the major difference in the wire region, an oscillatory feature due to the charge rearrangement appears on a few layers in each electrode, in good agreement with the previous calculations 17. Thus, the resonance states are not strictly localized in the wire but extended somewhat into the electrodes.

In the PDOS, there exists a half-filled resonance state at $E_{F}$ for odd $L$ (on-resonance), while the Fermi level lies between two resonance states for even $L$ (off-resonance). The position of the resonance states affects $N_{e}-N_{o}$, and thus conductances. For demonstration, we use the relation $N_{e}-N_{o}=\left(N_{e}-N_{e}^{\mathrm{R}}\right)-\left(N_{o}-N_{o}^{\mathrm{R}}\right)$, where $N_{e}^{\mathrm{R}}=N_{o}^{\mathrm{R}}$ because $G^{\mathrm{R}}=0$ in the reference system. Since each resonance contains exactly one extra energy level [18], each filled resonance state with even (odd) parity increases $N_{e(o)}-N_{e(o)}^{\mathrm{R}}$ by 2 without altering $N_{o(e)}-N_{o(e)}^{\mathrm{R}}$, and thus increases (decreases) $N_{e}-N_{o}$ by 2 ; the factor two denotes spin degeneracy. However, the filled resonance states are irrelevant because $G$ does not change when $N_{e}-N_{o}$ changes by \pm 2 , as shown in Eq. (11). On the other hand, the half-filled resonance state changes $N_{e}-N_{o}$ by \pm 1 , and if there is such a state, $G$ equals $G_{0}$. When the Fermi level is located at the tails of resonances, $\left|N_{e}-N_{o}\right|$ can be quite different from 1 and $G<G_{0}$. This explains the correlation between the even-odd behavior of conductances and the relative position of resonance states with respect to $E_{F}$.

A recent tight-binding study [6] for $L=1$ has demonstrated that the position of resonance states cannot be arbitrary when the local charge neutrality is assumed. Although this assumption is violated in atomic scale due to the charge oscillation in Fig. 1 (b), we find that the charge neutrality does hold globally near the wire and constrains the resonance position for general $L$. Since $\mathrm{Na}$ is a monovalent atom, the wire system contains $\Delta N$ $(=L-2)$ extra electrons, as compared to its reference system. On the other hand, we verify that the numbers of filled resonances in the two systems differ by $\Delta N / 2$ [19], which is a half-integer for odd $L$. This matching indicates that all extra electrons are in the resonance states near the wire. Thus, the alternation of on- and off-resonances with $L$ results from the charge neutrality.

The same even-odd behavior is expected for a wide class of wire structures, since the resonant character and the charge neutrality are common features. We examine various wire structures such as stretched wires with $d=1.1$ and $1.2 d_{0}$ and zigzag wires with the bond angles $\theta=150^{\circ}$ and $120^{\circ}\left(d=d_{0}\right)$ [see the inset in Fig. 1(a)]. In all cases, a half-filled resonance state is found for odd $L$ and $G=G_{0}$ to within $2 \%$, while the off-resonance transport is realized for even $L$ and $G<G_{0}$. Thus, the same even-odd behavior holds for other wire structures. On a quantitative level, however, $G$ is sensitive to the wire structure for even $L$. For the $(L=4, M=64)$ system, for example, $G / G_{0}$ is 0.61 for a linear unstretched wire, 0.50 for a stretched wire with $d=1.2 d_{0}$, and 0.71 for a zigzag wire with $\theta=120^{\circ}$. Recalling that in the off-resonance transport, $G$ depends not only on the relative position of resonance states but also on the ratio $\Gamma / \Delta E$, where $\Delta E$ and $\Gamma$ denote the resonance spacing and width, respectively, nonuniversal $G$ values for even $L$ can be understood since the ratio depends on wire structure. For sufficiently large $L$, however, $G$ becomes universal for even $L$ as well since $\Delta E \rightarrow 0$ and $G \rightarrow G_{0}$ as $L \rightarrow \infty$ [20]. In this limit, the even-odd behavior disappears.

Here we note that two key ingredients responsible for the even-odd behavior are the sharp tip structure and charge neutrality, which are believed to be common in other monovalent metallic wires. It is then very plausible that the same even-odd behavior may appear in other monovalent metallic wires. Previous experimental studies [1,21] showed that atomic contacts made of various monovalent metals have many properties in common. In agreement with our expectation, recent calculations [22] 
for gold wires found that $G$ is smaller than $G_{0}$ and sensitive to wire structure for $L=4$. Experimentally, gold wires with $L>1$ have been already reported [23,24. Although these experiments did not exhibit an evidence for the even-odd behavior, it is premature to reject the possibility of the even-odd behavior in gold wires because it is not clear whether uncontaminated gold wires with even and odd $L$ are both produced in the experiments [25]. More experimental studies are required to clarify the existence of the even-odd behavior.

We next discuss briefly atomic wires made of multivalent atoms. Since multiple eigenchannels contribute to transport, Eq. (11) should be replaced by the generalized formula [11], $G=G_{0} \sum_{j=1}^{J} \sin ^{2}\left[(\pi / 2)\left(N_{e}-N_{o}\right) \alpha_{j}\right]$, where $J$ is the number of channels and $\sum_{j=1}^{J} \alpha_{j}=1$. One crucial difference from monovalent wires is that the new parameter $\alpha_{j}$ does depend on wire structure. Thus, even when the charge neutrality fixes $N_{e}-N_{o}$, G still depends on wire structure. This explains why multivalent metallic wires such as Al [1] do not exhibit clearly quantized conductances as in monovalent wires. We point out that for $L=1$, the multichannel transport may be relevant even for sodium wires. We find that the coupling between two electrodes is not negligible even when the shared apex atom is removed. This implies that the direct transport between the electrodes, which is not mediated by the common apex atom, is not negligible, in agreement with Ref. 9]. For more precise conductance calculations for $L=1$, the generalized formula should be used, which is beyond the scope of our paper. In recent experiments for $\mathrm{Na}$ atom contacts [26], the tail of the conductance histogram peak at $G_{0}$ was shown to be extended up to $1.2 G_{0}$, which may be interpreted as an indication of at least two additional eigenchannels 27] with a small contribution of $\sim 0.1 G_{0}$ for each channel.

In conductance calculations using finite-sized electrodes, the level broadening $E_{T}$ should satisfy the following constraints; to retain the metallic nature, $E_{T}$ should be larger than single particle level spacing $\delta \epsilon(\sim 0.02$ $\mathrm{eV}$ near $E_{F}$ ), while it should be smaller than $\Gamma$ and $\Delta E$ $(\sim 0.3 \mathrm{eV})$. When $E_{T}$ varies between 0.03 and $0.15 \mathrm{eV}$, we verify that $G$ for odd $L$ is almost independent (to within a few percent). For even $L$, on the other hand, the resonance width $\Gamma$ depends weakly on $E_{T}$ and thus the choice of $E_{T}$ affects $G$ quantitatively although the even-odd behavior remains robust. We also point out that $\Gamma$ depends on the sharpness of the tip structure. For the pyramidshape (100) tip, which is less sharper than the (111) tip, we find that $\Gamma$ increases by about $40 \%$ and the overlap of resonances is enhanced, leading to weaker even-odd behavior and less accurate conductance quantization for odd $L$. Recent calculations [9] for an $L=3$ wire with flat electrodes showed that the energy dependence of the transmission coefficient is almost negligible, implying an even larger $\Gamma$ of about $1 \mathrm{eV}$.
Finally, we note that when the exact inversion (or mirror reflection) symmetry is relaxed, the even-odd behavior still occurs because the on(off)-resonance for odd (even) $L$ arises from the sharp tip structure and charge neutrality. However, the conductance quantization for odd $L$ is weakened, as illustrated clearly in Ref. [6]. Recent experiments [28] have demonstrated that the electrodes of atomic contacts tend to be aligned with symmetric lattice axes. Here we remark that a similar conductance oscillation due to the resonance states is realized for heterogeneous systems [29], where wires and electrodes are made of different atomic elements. The origin of the resonance is however different; in heterogeneous systems, the charge transfer between the wires and electrodes is likely to occur and the resulting Schottkylike potential barriers at both the wire ends generate the resonance states even if the electrodes are flat.

In summary, a first-principles method is implemented to calculate conductances through $\mathrm{Na}$ wires, with the aid of the Friedel sum rule. Unlike conventional QPCs, the transport in monatomic $\mathrm{Na}$ wires has resonant character due to the sharp tip structure. Combined with the charge neutrality, the resonance states lead to robust quantized conductances when the number $(L)$ of atoms in the wire is an odd number. For even $L$, the off-resonance transport is realized with $G$ smaller than $G_{0}$. We suggest that the same even-odd behavior of conductances may appear in other monovalent atomic wires.

We thank Y.-G. Jin for his advice on the efficient realspace multigrid method. We also acknowledge helpful discussions with profs. C. S. Kim and J. Yu. This work was supported by the QSRC at Dongkuk University.

[1] J. M. van Ruitenbeek, cond-mat/9910394.

[2] L. Olesen et al., Phys. Rev. Lett. 72, 2251 (1994).

[3] B. J. van Wees et al., Phys. Rev. Lett. 60, 848 (1988); D. A. Wharam et al., J. Phys. C 21, L209 (1988).

[4] L. I. Glazman et al., JETP Lett. 48, 238 (1988).

[5] J. M. Krans et al., Phys. Rev. Lett. 74, 2146 (1995).

[6] A. Levy Yeyati, A. Martín-Rodero, and F. Flores, Phys. Rev. B 56, 10369 (1997); J. C. Cuevas et al., Phys. Rev. Lett. 81, 2990 (1998).

[7] E. G. Emberly and G. Kirczenow, Phys. Rev. B 60, 6028 (1999).

[8] M. Brandbyge, N. Kobayashi, and M. Tsukada, Phys. Rev. B 60, 17064 (1999).

[9] N. Kobayashi, M. Brandbyge, and M. Tsukada, Surf. Sci. 433-435, 854 (1999); Phys. Rev. B 62, 8430 (2000).

[10] Monatomic Na wires with $L>1$ [Fig. 11(a)] have not been reported experimentally yet, while a possibility of 3 atom-long $\mathrm{Na}$ wires has been demonstrated theoretically; A. Nakamura et al., Phys. Rev. Lett. 82, 1538 (1999).

[11] S. Datta and W. Tian, Phys. Rev. B 55, R1914 (1997). 
[12] H.-W. Lee, Phys. Rev. Lett. 82, 2358 (1999).

[13] H. E. van den Brom and J. M. van Ruitenbeek, Phys. Rev. Lett. 82, 1526 (1999).

[14] Y.-G. Jin, J.-W. Jeong, and K. J. Chang, Physica B 273274, 1003 (1999).

[15] N. Troullier and J. L. Martins, Phys. Rev. B 43, 1993 (1991); L. Kleinman and D. M. Bylander, Phys. Rev. Lett. 48, 1425 (1982).

[16] M. C. Payne et al., Rev. Mod. Phys. 64, 1045 (1992).

[17] V. A. Sablikov, S. V. Polyakov, and M. Büttiker, Phys. Rev. B 61, 13763 (2000).

[18] See for instance W. A. Harrison, Solid State Theory, Fig. 2.47 (Dover, New York, 1979).

[19] We remark that the reference system itself has doubly degenerate (one with odd and the other with even parity) resonance states (near black circles in Fig. 3), which are mostly localized near the apex atoms of the electrodes. When the two electrodes are connected to make, for example, an $L=5$ wire, the doubly degenerate resonance states near $E_{F}-0.3 \mathrm{eV}$ are mixed with the wire states to generate three filled and one half-filled resonance states. Then, the number of new filled resonance states is $1 \frac{1}{2}$.

[20] H.-W. Lee and C. S. Kim, Phys. Rev. B 63, 075306 (2001); J. Korean Phys. Soc. 37, 137 (2000).

[21] E. Scheer et al., Nature (London) 394, 154 (1998).

[22] See Ref. [12] in H. Häkkinen, R. N. Barnett, and U. Landman, J. Phys. Chem. B 103, 8814 (1999).

[23] A. I. Yanson et al., Nature (London) 395, 783 (1998).

[24] H. Ohnishi, Y. Kondo, and K. Takayanagi, Nature (London) 395, 780 (1998).

[25] For rotating zigzag gold wires proposed by D. SánchezPortal et al. [Phys. Rev. Lett. 83, 3884 (1999)], L should be an odd number to be consistent with the large interatomic spacing observed in Ref. 24. In addition, there may be a possibility that gold wires are contaminated by atoms with low atomic numbers.

[26] J. M. Krans et al., Nature (London) 375, 767 (1995).

[27] With only one additional channel, $G$ is still $G_{0}$ due to the identity relation, $\sin ^{2}[(\pi / 2) \alpha]+\sin ^{2}[(\pi / 2)(1-\alpha)]=1$.

[28] V. Rodrigues et al., Phys. Rev. Lett. 85, 4124 (2000).

[29] N. D. Lang, Phys. Rev. Lett. 79, 1357 (1997); N. D. Lang and Ph. Avouris, Phys. Rev. Lett. 84, 358 (2000).

(a)
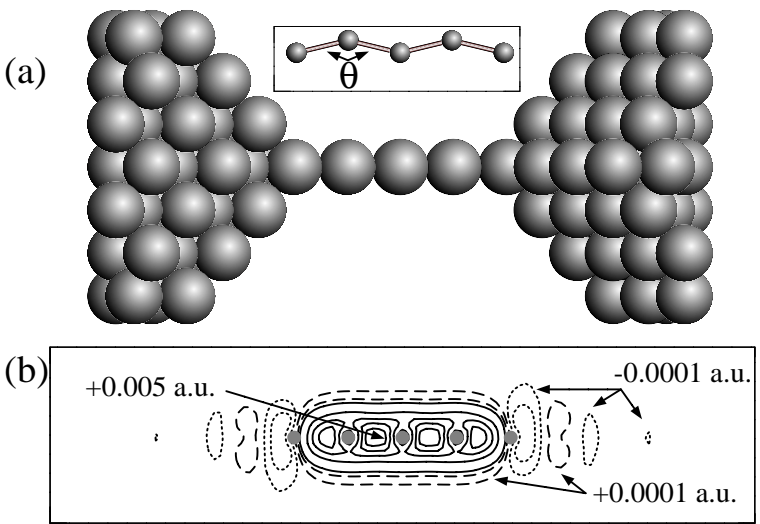

FIG. 1. (a) The atomic structure for the $L=5 \mathrm{Na}$ wire connected to electrodes, with the inversion symmetry. Each electrode is modeled by a cluster of $M(=64) \mathrm{Na}$ atoms. (b) Contour plot of the difference of the total charge densities between the ( $L=5, M=64)$ wire and its reference system: solid contours for 0.001 to 0.005 a.u. with the increment of 0.001 a.u., whereas dotted and dashed contours for -0.0003 to 0.0003 a.u. with the increment of 0.0002 a.u. Atomic positions in the wire are marked by gray dots. A zigzag wire is shown in the inset in (a).

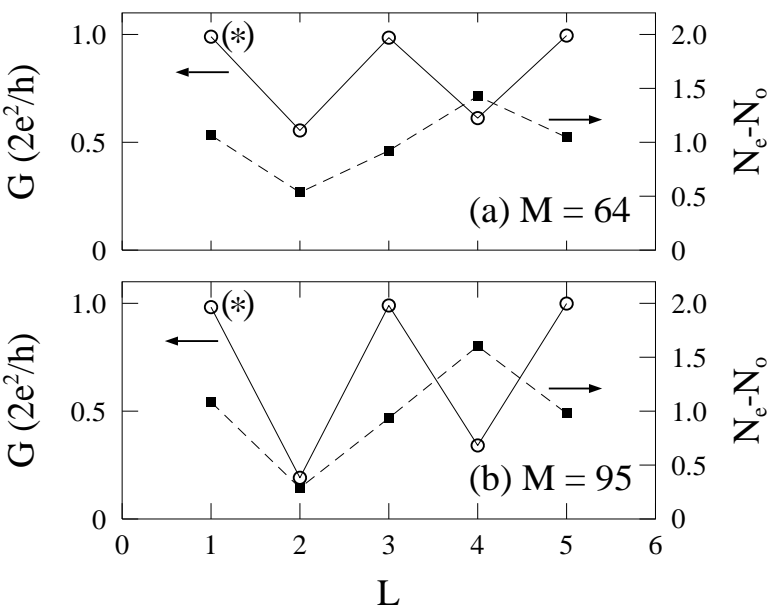

FIG. 2. For unstretched linear wires with (a) $M=64$ and (b) $95, G(L)$ (open circles) and $N_{e}-N_{o}$ (filled squares) are plotted as a function of $L$. For $G(1)$ marked by asterisks, its value may be modified due to the possibility of multi-channel transport (see text).

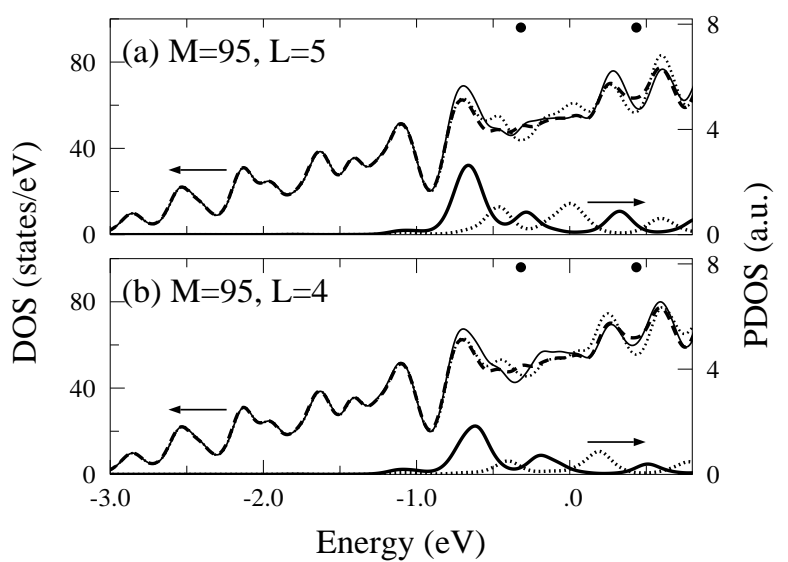

FIG. 3. For a linear wire with $d=d_{0}, \rho_{e}(E)$ (solid lines) and $\rho_{o}(E)$ (dotted lines) are compared with $\rho_{e}^{\mathrm{R}}(E)\left[=\rho_{o}^{\mathrm{R}}(E)\right]$ (dashed lines). The Fermi level $\left(E_{F}\right)$ is aligned at $0 \mathrm{eV}$. Black circles denote the locations of the resonance states which are localized at the apex atoms of the reference system. Thick solid and dotted lines denote the projected DOSs (PDOSs) with even and odd parities, respectively. 